\title{
A candidate protostellar object in the L 1457/MBM 12 cloud
}

\author{
A. Heithausen ${ }^{1}$ and C. Böttner ${ }^{2}$ \\ 1 Institut für integrierte Naturwissenschaften, Abteilung Physik, Universität Koblenz-Landau, Universitätsstr. 1, 56070 Koblenz, \\ Germany \\ e-mail: heithausen@uni-koblenz.de \\ 2 Radioastronomisches Institut, Universität Bonn, Auf dem Hügel 71, 53121 Bonn, Germany \\ e-mail: christoph-boettner@gmx.de \\ Received 3 March 2010 / Accepted 30 September 2010
}

\section{ABSTRACT}

\begin{abstract}
Aims. The association of young T Tauri stars, MBM 12A, indicates that L 1457 was forming stars not too long ago. With our study we want to find out whether there are still signs of ongoing star formation in that cloud.

Methods. Using the Max-Planck-Millimeter-Bolometer MAMBO at the IRAM $30 \mathrm{~m}$ telescope, we obtained a map of about $8^{\prime} \times 8^{\prime}$ centered on $\mathrm{L} 1457$ in the dust continuum emission at $230 \mathrm{GHz}$. Towards the most intense regions in our bolometer map, we obtained spectra at high angular resolution in the $\mathrm{CS}(2 \rightarrow 1)$ and the $\mathrm{N}_{2} \mathrm{H}^{+}(1 \rightarrow 0)$ lines using the IRAM $30 \mathrm{~m}$ telescope.

Results. We find that the cold dust in L 1457 is concentrated in several small cores with high $\mathrm{H}_{2}$ column densities and solar masses. The density profiles of the cores are inconsistent with a sphere with constant density. These cores are closer to virial equilibrium than is the cloud as a whole. Data from the VLA and Spitzer archives reveal two point sources in the direction of one dust core. One of the sources is probably a distant quasar, whereas the other source is projected right on a local maximum of our dust map and shows characteristics of a protostellar object.
\end{abstract}

Key words. ISM: clouds - ISM: abundances - ISM: molecules - stars: formation

\section{Introduction}

Class 0 objects are protostellar objects at the beginning of the accretion phase, where the bulk of the final stellar material has not been assembled yet (André et al. 1993). These objects have been detected towards various regions with known ongoing star formation (André et al. 1993, 1999; Kauffmann et al. 2005). Molecular cirrus clouds at high Galactic latitudes so far show only very little star-formation activity, no embedded infrared sources had been found in the IRAS faint source catalog (Magnani et al. 1995). Nevertheless, the existence of a gravitationally bound core in a cirrus clouds (Heithausen et al. 2002, 2008), which shows evidence of inward motion (Heithausen 1999), shows that these clouds harbor potential sites of star formation.

One candidate for possible star formation in such clouds is the dark cloud L 1457, located at southern Galactic latitudes below the Taurus region. This cloud is puzzling for two reasons:

- complete CO maps of L 1457 (Magnani et al. 1985; Zimmermann \& Ungerechts 1990; Pound et al. 1990) indicate that the cloud as a whole is far from being gravitationally bound. Reach et al. (1995) found extensive CS emission throughout the cloud, indicating dense molecular gas that is closer to virial equilibrium;

- several T Tauri stars have been detected in the direction of L 1457 (Hearty et al. 2000; Luhman 2001), forming the association MBM 12A (see Fig. 1). For this assocation, Luhman (2001) determined an age of about 2 million years, indicating that, if the young stars are indeed associated with the cloud, L 1457 was forming stars not too long ago.

To shed light on its star-forming capability, we obtained a bolometer map of the densest part of the L 1457/MBM 12 cloud at a wavelength of $1.2 \mathrm{~mm}$ with the IRAM $30 \mathrm{~m}$ telescope. The selected region was found to be the most intense portion in the high angular resolution data of the ${ }^{12} \mathrm{CO}$ and ${ }^{13} \mathrm{CO}(1 \rightarrow 0)$, $(2 \rightarrow 1)$, and $(3 \rightarrow 2)$ transitions obtained by Zimmermann (1993). Supplementary information on the dynamical state of the core comes from CS $(2 \rightarrow 1)$ observations obtained with the IRAM $30 \mathrm{~m}$ telescope, too. We discuss a possible association of two continuum point sources detected with the VLA at a wavelength of $3.6 \mathrm{~cm}$ and with Spitzer Space Telescope at $24 \mu \mathrm{m}$.

\section{Observations}

L 1457 was observed in 2000 and 2001 in the dust continuum at $1.2 \mathrm{~mm}$ using the Max-Planck-Millimeter-Bolometer MAMBO at the IRAM $30 \mathrm{~m}$ radio telescope on Pico Veleta, Spain. MAMBO is sensitive to emission between 210 and $290 \mathrm{GHz}$, with an effective frequency of $250 \mathrm{GHz}$ for steep thermal spectra. The observations were taken in double-beam onthe-fly mode, i.e., chopping the secondary mirror in azimuth by $50^{\prime \prime}$ to $70^{\prime \prime}$ at $2 \mathrm{~Hz}$ and scanning the sky in azimuth at a speed of $4^{\prime \prime}$ to $5^{\prime \prime} \mathrm{s}^{-1}$, then moving in elevation by $4^{\prime \prime}$. The maps were taken under variable winter conditions with line-of-sight opacities between 0.2 and 0.7 . The effective beam FWHM is $11^{\prime \prime}$.

The most intense dust positions in L 1457 were oberved in the CS $(2 \rightarrow 1)$ transition in October 2002 and in the $\mathrm{N}_{2} \mathrm{H}^{+}(1 \rightarrow$ 0) transition in August 2004 with the IRAM 30 m radiotelescope. Data were taken in single-position on-off-mode. We obtained small maps with $20^{\prime \prime}$ spacing between individual positions. We used the VESPA autocorrelation spectrometer with a velocity resolution set to $0.062 \mathrm{~km} \mathrm{~s}^{-1}$ at $93 \mathrm{GHz}$ and $0.060 \mathrm{~km} \mathrm{~s}^{-1}$ at $98 \mathrm{GHz}$. The angular resolution of the $30 \mathrm{~m}$ telescope at $93 \mathrm{GHz}$ and $98 \mathrm{GHz}$ is $27^{\prime \prime}$ and $26^{\prime \prime}$, respectively. The main beam efficiency $\eta_{\mathrm{mb}}=0.8$. 

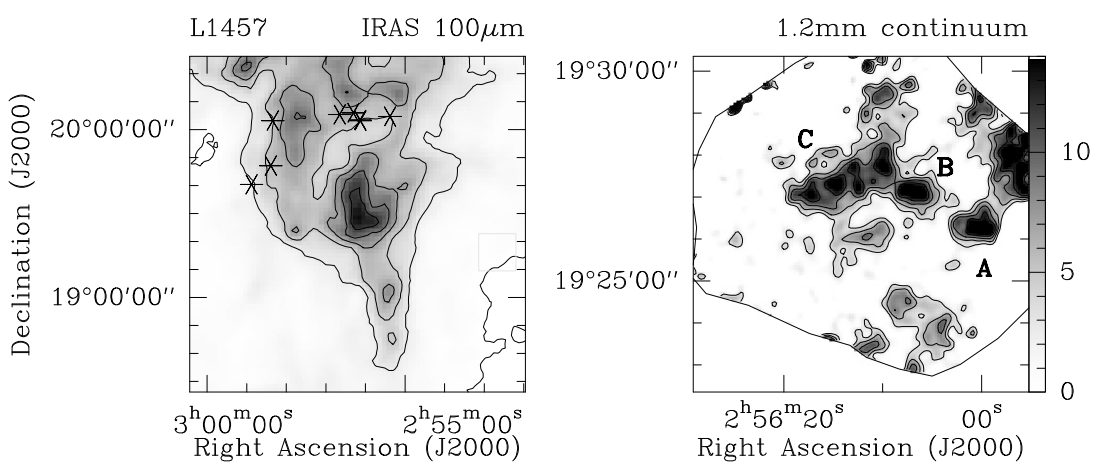

Fig. 1. IRAS $100 \mu \mathrm{m}$ and MAMBO $1.2 \mathrm{~mm}$ maps of L 1457. In the IRAS map contour lines are every $5 \mathrm{MJy} / \mathrm{sr}$ starting at $5 \mathrm{MJy} / \mathrm{sr}$. The position of our CS and bolometer maps is marked by the white box. The asterisks mark the positions of the T Tauri stars in the L 1457/MBM 12 region described by Luhman (2001). Contours in the MAMBO $1.2 \mathrm{~mm}$ dust emission map are every $3 \mathrm{mJy} /$ beam starting at $3 \mathrm{mJy} /$ beam $(3 \sigma)$. The positions of the three condensations in the dust map are labeled A, B, and C.

Table 1. $1.2 \mathrm{~mm}$ sources in L 1457 .

\begin{tabular}{llllllllll}
\hline \hline Source & $\begin{array}{l}\text { RA } \\
(\mathrm{J} 2000)\end{array}$ & $\begin{array}{l}\text { Dec. } \\
(\mathrm{J} 2000)\end{array}$ & $\begin{array}{l}\text { Size } \\
\left({ }^{\prime \prime} \times{ }^{\prime \prime}\right)\end{array}$ & $\begin{array}{l}\text { Radius } \\
(\mathrm{pc})\end{array}$ & $\begin{array}{l}\text { Peak } F_{v}^{\text {beam }} \\
(\mathrm{mJy} / \mathrm{beam})\end{array}$ & $\begin{array}{l}\text { Peak } N_{\mathrm{H}_{2}} \\
\left(\mathrm{~cm}^{-2}\right)\end{array}$ & $\begin{array}{l}F_{v} \\
(\mathrm{Jy})\end{array}$ & $\begin{array}{l}M_{\mathrm{H}_{2}} \\
\left(M_{\odot}\right)\end{array}$ & $\begin{array}{l}\text { Virial mass } \\
\left(M_{\odot}\right)\end{array}$ \\
\hline L 1457-A & $2: 56: 00.6$ & $19: 26: 17$ & $67 \times 46$ & 0.07 & $20 \pm 1$ & $1.3 \times 10^{22}$ & $0.23 \pm 0.03$ & 1.0 & $\leq 3$ \\
L 1457-B & $2: 56: 07.0$ & $19: 27: 10$ & $57 \times 40$ & 0.06 & $17 \pm 1$ & $1.1 \times 10^{22}$ & $0.17 \pm 0.02$ & 0.7 & $\leq 4-10$ \\
L 1457-C & $2: 56: 13.5$ & $19: 27: 30$ & $146 \times 56$ & 0.11 & $17 \pm 1$ & $1.1 \times 10^{22}$ & $0.74 \pm 0.05$ & 3.1 & $\leq 5$ \\
L 1457-C1 & $2: 56: 17.2$ & $19: 27: 04$ & $54 \times 55$ & 0.08 & $17 \pm 1$ & $1.1 \times 10^{22}$ & $0.21 \pm 0.03$ & 0.9 & $\leq 3$ \\
\hline
\end{tabular}

Additionally, we used public data from the VLA and Spitzer archives. In the VLA archive we found two thus far unpublished maps centered on about the same position as our bolometer map. The data were observed on March 6 and 25, 1991 at a wavelength of $3.55 \mathrm{~cm}$ with an angular resolution of $10^{\prime \prime}$. The primary beam of the VLA has a full width at half maximum of 5.4 and covers all our bolometer sources (see Fig. 3). The noise level in the VLA map is $0.03 \mathrm{mJy} / \mathrm{beam}$. Part of the region was also observed with MIPS (Rieke et al. 2004) onboard the Spitzer Space Telescope (Werner et al. 2004) at a wavelength of $24 \mu \mathrm{m}$. The data were observed on September 23, 2007. The angular resolution of the MIPS instrument at that wavelength is about $6^{\prime \prime}$, map spacing is $2.5^{\prime \prime}$. The data taken from the Spitzer archive were already calibrated using the standard pipeline. The noise level in the MIPS map is $0.06 \mathrm{MJy} / \mathrm{sr}$.

\section{The distribution of cold dust}

Our MAMBO $1.2 \mathrm{~mm}$ dust map is presented in Fig. 1. Dust is concentrated in several small condensations. A possible extensive diffuse dust component is filtered out by our observing technique. We labeled the three most prominent structures as L 1457-A, B, and C. The part of the core where we detected a point source (see Sect. 4) is referred to as L1457-C1.

To calculate the molecular hydrogen column density $N_{\mathrm{H}_{2}}$ from the peak flux density $F_{v}^{\text {beam }}$ we use the formula provided by Kauffmann et al. (2008), who adopted a dust temperature of $10 \mathrm{~K}$ and a wavelength of $1200 \mu \mathrm{m}$ :

$N_{\mathrm{H}_{2}}=6.69 \times 10^{20} \mathrm{~cm}^{-2}\left(\frac{F_{v}^{\text {beam }}}{\text { mJy beam }^{-1}}\right)$.

Ammonia observations reveal a gas temperature of about $T_{\text {kin }} \leq$ $12 \mathrm{~K}$ for the dense core (Gomez et al. 2000). We derive molecular hydrogen column density of up to $N_{\mathrm{H}_{2}}=1.3 \times 10^{22} \mathrm{~cm}^{-2}$ (see Table 1). Towards XY Ari, a star in the middle of our field, Littlefair et al. (2001) derived a visual extinction of $A_{V}=11.5 \pm 0.3 \mathrm{mag}$, confirming our estimate of the molecular hydrogen column density.

Kauffmann et al. (2008) also provide a formula for deriving the molecular hydrogen mass $M_{\mathrm{H}_{2}}$ from the total bolometer flux
$F_{v}$ at a wavelength of $1200 \mu \mathrm{m}$ for an object with $10 \mathrm{~K}$ temperature which is applicable to our observations

$$
M_{\mathrm{H}_{2}}=0.47 M_{\odot}\left(\frac{F_{v}}{\mathrm{Jy}}\right)\left(\frac{d}{100 \mathrm{pc}}\right)^{2}
$$

where $d$ is the distance to the cloud. With a distance of $d=65 \mathrm{pc}$, L 1457 has long been thought to be the nearest molecular cloud (Hobbs et al. 1986). Recent distance determinations suggest, however, a much higher distance. Andersson et al. (2002) estimate a distance of $360 \pm 30$ pc to L 1457; Luhman (2001) finds $275 \mathrm{pc}$, and Straižys et al. (2002) find $325 \mathrm{pc}$. Adopting a distance of $300 \mathrm{pc}$ to the cloud, we find masses in the range of $0.7 M_{\odot} \leq M_{\mathrm{H}_{2}} \leq 3.1 M_{\odot}$ (Table 1$)$.

Similar to our approach for MCLD123.5+24.9 (Heithausen et al. 2008) we tried to determine the density profiles of L1457-A and $\mathrm{B}$ by obtaining circular averages of the intensity distributions centered on the cores. We find that both are inconsistent with spheres of constant volume densities. Better fits can be derived by Gaussian profiles or centrally peaked density profiles similar to those given by Johnstone et al. (2003, see also Heithausen et al. 2008).

To analyze the stability of the cores we compare their masses with their virial masses $M_{\mathrm{vir}}=3 r \sigma^{2} / G$, where $\sigma=\Delta v / 2.355$ is the one-dimensional gas velocity dispersion, $r$ the radius of the cloud, and $G$ the gravitational constant. Here we assume a density profile proportional to $r^{-2}$. Because towards the regions with significant dust emission no $\mathrm{N}_{2} \mathrm{H}^{+}$was detected down to a rms of $\leq 0.06 \mathrm{~K}\left(T_{\mathrm{mb}}\right)$ in $0.06 \mathrm{~km} \mathrm{~s}^{-1}$ wide channels, we use CS $(2 \rightarrow 1)$ data obtained at high angular resolution with the IRAM $30 \mathrm{~m}$ telescope (s. Fig. 2) to estimate the velocity dispersion. Parameters of a Gaussian analysis of the CS spectra are listed in Table 2. For L 1457-B and C, the CS spectra show multiple components. For our estimate of the virial mass we took the components closest to $v_{\mathrm{LSR}}=-5 \mathrm{~km} \mathrm{~s}^{-1}$, which is the velocity of the main CS component. If we correct for the contribution of Helium, the ratio of cloud mass to virial mass is in the range of 0.2 to 0.6 , indicating that the cores are close to virial equilibrium. This ratio is a lower limit because CS traces a larger volume in the core (e.g. Reach et al. 1995) than our bolometer sources Furthermore the CS line might be optically thick. 

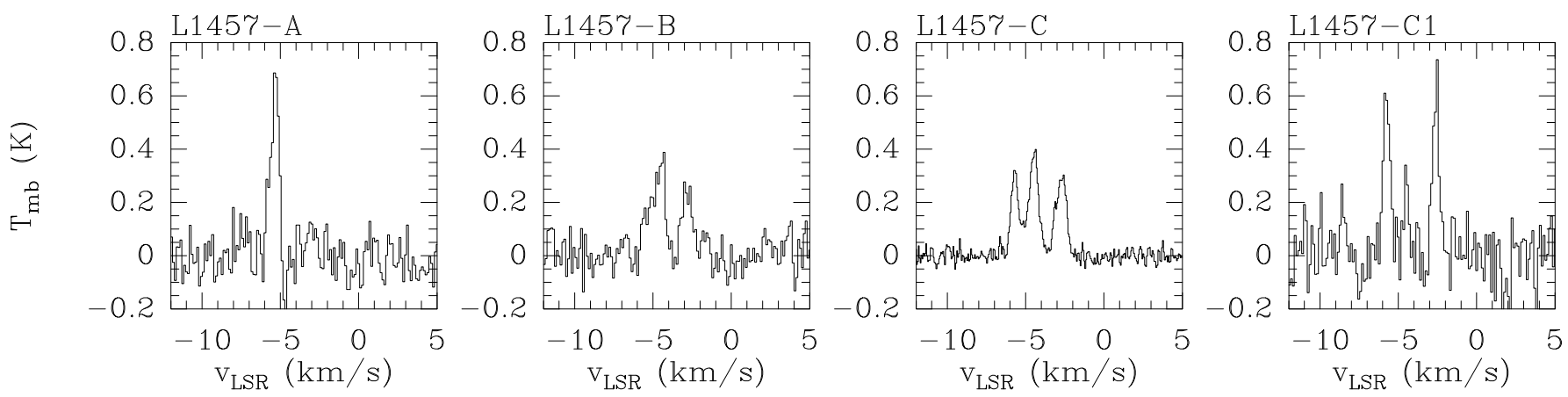

Fig. 2. CS spectra towards the L 1457-A, L 1457-B, and L 1457-C, as obtained with the IRAM $30 \mathrm{~m}$ telescope. For L 1457-A and B, we only obtained single spectra towards the center of the cores. For L 1457-C we obtained a large map with 50 individual positions, which we averaged. The rightmost spectrum shows a single CS spectrum towards the position of J025616+192703.
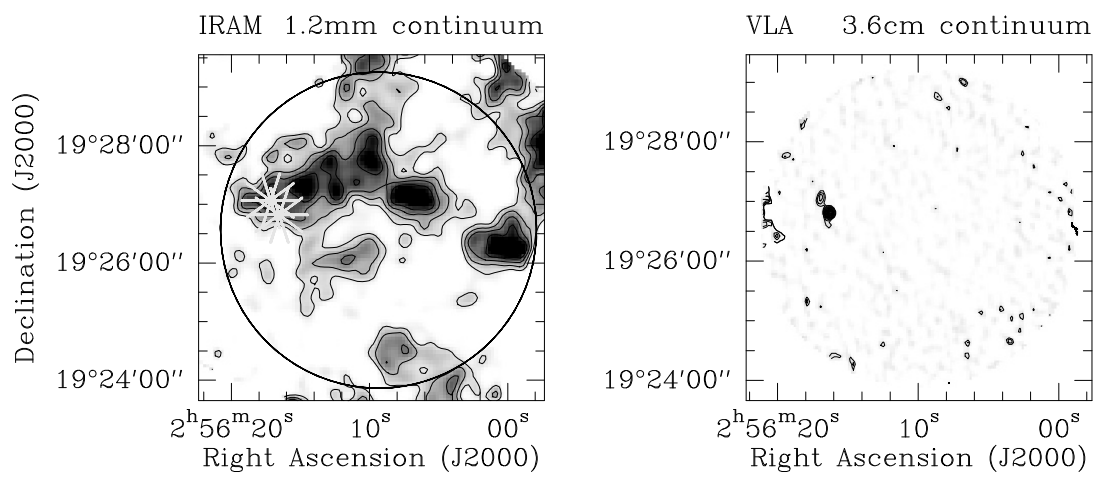

Fig. 3. Comparison of the VLA map (right) at $\lambda=$ $3.55 \mathrm{~cm}$ and our bolometer map (left). The circle in the bolometer map indicates the primary beam of the VLA map. The positions of the VLA sources are marked by asterisks. Contours in the bolometer map are the same as in Fig. 1. Contours in the VLA map are every $0.07 \mathrm{mJy} /$ beam starting at $0.07 \mathrm{mJy} /$ beam (2 sigma).

Table 2. Parameters of the IRAM CS observation in L 1457.

\begin{tabular}{lllll}
\hline \hline Source & $\begin{array}{l}T_{\mathrm{mb}} \\
(\mathrm{K})\end{array}$ & $\begin{array}{l}\mathrm{rms} \\
(\mathrm{K})\end{array}$ & $\begin{array}{l}v_{\mathrm{LSR}} \\
\left(\mathrm{km} \mathrm{s}^{-1}\right)\end{array}$ & $\begin{array}{l}\Delta v \\
\left(\mathrm{~km} \mathrm{~s}^{-1}\right)\end{array}$ \\
\hline L 1457-A & 0.69 & 0.09 & $-5.35 \pm 0.02$ & $0.56 \pm 0.04$ \\
L 1457-B & 0.31 & 0.08 & $-4.71 \pm 0.05$ & $1.17 \pm 0.11$ \\
& 0.25 & 0.08 & $-2.82 \pm 0.05$ & $0.74 \pm 0.10$ \\
L 1457-C & 0.37 & 0.02 & $-5.69 \pm 0.01$ & $0.61 \pm 0.03$ \\
& 0.31 & 0.02 & $-4.49 \pm 0.01$ & $0.74 \pm 0.03$ \\
& 0.30 & 0.02 & $-2.74 \pm 0.01$ & $0.79 \pm 0.03$ \\
L 1457-C1 & 0.62 & 0.10 & $-5.75 \pm 0.03$ & $0.50 \pm 0.06$ \\
& 0.33 & 0.10 & $-4.49 \pm 0.04$ & $0.31 \pm 0.11$ \\
& 0.64 & 0.10 & $-2.59 \pm 0.03$ & $0.48 \pm 0.06$ \\
\hline
\end{tabular}

We might therefore systematically overestimate the line width, hence also the virial mass.

\section{Radio and infrared sources towards the core of L 1457/MBM 12}

The VLA $3.6 \mathrm{~cm}$ maps (Fig. 3) show two point sources. The brighter one has already been detected by Gomez et al. (2000) from other VLA archive data and they named it VLA B0253+192. About $18^{\prime \prime}$ to the northeast we detected a further point source, which we name J025616+192703. Positions and fluxes are listed in Table 3.

For VLA B0253+192, Gomez et al. (2000) give a flux density of $S_{8.4 \mathrm{GHz}}=0.114 \pm 0.026 \mathrm{mJy}$ at the observed wavelength. We determine a higher flux $S_{8.4 \mathrm{GHz}}=0.60 \pm 0.04 \mathrm{mJy}$, so the source might be variable. The source is also listed in the NVSS catalog by Condon et al. (1998) at a frequency of $1.4 \mathrm{GHz}$. At that frequency Condon et al. give a flux density of $S_{1.4 \mathrm{GHz}}=$ $3.4 \mathrm{mJy}$. Using the flux density by Gomez et al. (2000) we find a spectral index of $\alpha_{\text {Radio }}=-1.9$, while we find $\alpha_{\text {Radio }}=$ -1.0 using our value. Both values indicate a steep radio spectrum typical for non-thermal emission. This confirms the proposal by Gomez et al. that the source might be an unrelated background source, possibly a distant quasar.

For J025616+192703 no counterpart can be found in the NVSS catalog. Due to the angular resolution of NVSS catalog of 45" (Condon et al. 1998), this source is at only half a beam distance from VLA B0253+192. To separate a possible contribution of J025616+192703, we subtracted the emission of VLA B0253+192 from the NVSS image adopting a circular beam size. This way we find an upper limit of $0.3 \mathrm{mJy}$ for the flux of J025616+192703 at $1420 \mathrm{MHz}$. The spectral index derived with that value would close to or larger than zero, thus synchrotron radiation can be excluded. The spectrum is thus clearly different from that of VLA B0253+192, making a physical association between both sources unlikely.

Both sources are also visible in the Spitzer $24 \mu \mathrm{m}$ map (see Fig. 4). Fluxes and positions are given in Table 3. The Spitzer maps at $70 \mu \mathrm{m}$ and $170 \mu \mathrm{m}$ are unfortunately disturbed and cannot be used to determine the infrared spectra of the sources. In the IRAS $100 \mu \mathrm{m}$ map and our bolometer map the emission is extensive, so we can only determine upper limits for the source fluxes at those wavelengths.

As can be seen in Fig. 3 the source J025616+192703 is projected precisely on a local emission maximum in our bolometer map of L 1457-C. A chance alignment of a background source is very low. It is therefore possible that this source is indeed associated with the dense core, possibly a protostellar condensation, as a Class 0 object, deeply embedded in the core.

The few points of the spectrum of J025616+192703 that we know so far are consistent with established Class 0 sources. For example, IRAM 04191+1522 (André et al. 1999) would have a similar infrared intensity at $24 \mu \mathrm{m}$ (Dunham et al. 2006), if put at 


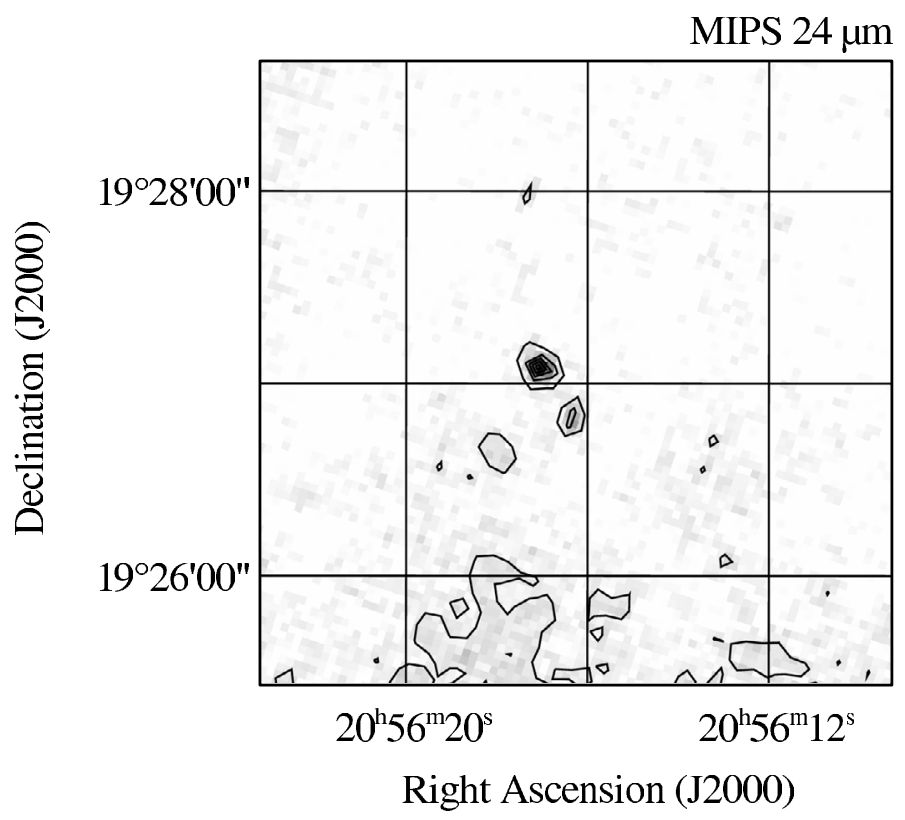

Fig. 4. $24 \mu \mathrm{m}$ Spitzer map of the dense core in L 1457. Contours are every $0.2 \mathrm{MJy} / \mathrm{sr}$. Note that the declination and right ascension axes are slightly rotated, as indicated by the grid.

Table 3. Parameters of the VLA and MIPS sources in L 1457.

\begin{tabular}{lll}
\hline \hline & J025616+192703 & VLA B0253+192 \\
\hline VLA 21 cm $\mathrm{c}^{\mathrm{I}}$ & & $2^{\mathrm{h}} 56^{\mathrm{m}} 16^{\mathrm{s}} .0$ \\
$\alpha(\mathrm{J} 2000)$ & - & $19^{\circ} 26^{\prime} 50^{\prime} 8$ \\
$\delta(\mathrm{J} 2000)$ & - & $3.4 \pm 0.5 \mathrm{mJy}$ \\
$S_{1.4 \mathrm{GHz}}$ & $\leq 0.3 \mathrm{mJy}$ & \\
VLA $3.6 \mathrm{~cm}$ & & \\
$\alpha(\mathrm{J} 2000)$ & $2^{\mathrm{h}} 56^{\mathrm{m}} 16^{\mathrm{s}} .9$ & $2^{\mathrm{h}} 56^{\mathrm{m}} 16^{\mathrm{s}} .3$ \\
$\delta(\mathrm{J} 2000)$ & $19^{\circ} 27^{\prime} 03^{\prime \prime} 6$ & $19^{\circ} 26^{\prime} 48^{\prime \prime} 8$ \\
$S_{8.4 \mathrm{GHz}}$ & $0.24 \pm 0.04 \mathrm{mJy}$ & $0.60 \pm 0.04 \mathrm{mJy}$ \\
MIPS $24 \mu \mathrm{m}$ & & \\
$\alpha(\mathrm{J} 2000)$ & $2^{\mathrm{h}} 56^{\mathrm{m}} 17^{\mathrm{s}} .0$ & $2^{\mathrm{h}} 56^{\mathrm{m}} 16^{\mathrm{s}} .3$ \\
$\delta(\mathrm{J} 2000)$ & $19^{\circ} 27^{\prime} 03^{\prime \prime} \cdot 9$ & $19^{\circ} 26^{\prime} 48^{\prime \prime} 6$ \\
$S_{24 \mu \mathrm{m}}$ & $2.0 \pm 0.2 \mathrm{mJy}$ & $1.0 \pm 0.2 \mathrm{mJy}$ \\
\hline
\end{tabular}

Notes. Remarks: 1: Condon et al. (1998).

the same distance. This source is also seen at radio wavelength with a similar flux (André et al. 1999). Recently, Kauffmann et al. (2005) and Bourke et al. (2006) have detected protostellar objects with very low luminosities. These sources cannot be distinguished from the extensive dust emission at mm-wavelength, but are more easily detected at shorter wavelengths, similar to J025616+192703. Without further points in the spectrum it is, however, not possible to draw more conclusions about the evolutionary state about our source.

\section{Summary and conclusions}

We have presented a bolometer map at $1.2 \mathrm{~mm}$, which shows at least three dense dust condensations with peak $\mathrm{H}_{2}$ column densities of $\geq 10^{22} \mathrm{~cm}^{-2}$ and solar masses. These are closer to virial equilibrium than is the source as a whole.

Towards one of the condensations, we find two point sources seen in the radio regime at $3.6 \mathrm{~cm}$ and in the infrared at $24 \mu \mathrm{m}$. The radio spectral index of one of the sources indicates nonthermal emission, thus the source is most likely a background object. For the other source the spectrum is only sparsely known. The exact location of the source at a local maximum in L 1457C suggests a physical assocation with the cloud. We speculate that this source could be a protostellar condensation that is still deeply embedded in the core. Its low luminosity and the detection only at radio and infrared wavelengths could be caused by an object with a temperature similar to that of the surrounding material.

To support this hypothesis more observations of the spectrum of this object between radio and infrared wavelength or a search for an associated outflow are necessary. Possibly owing to the multiple velocity components in the CO data of L1457 (Zimmermann 1993) no such outflow has been detected so far. If the protostellar nature of the source is confirmed, the study of this source will significantly increase our knowledge of lowmass star formation outside of the Galactic plane.

Acknowledgements. This work is based on observations carried out with the IRAM $30 \mathrm{~m}$ telescope and the VLA. IRAM is supported by INSU/CNRS (France), MPG (Germany), and IGN (Spain). The National Radio Astronomy Observatory is a facility of the National Science Foundation operated under cooperative agreement by Associated Universities, Inc. This work is also based in part on archival data obtained with the Spitzer Space Telescope, which is operated by the Jet Propulsion Laboratory, California Institute of Technology under a contract with NASA.

\section{References}

Andersson, B. G., Idzi, R., Uomoto, A., et al. 2002, AJ, 124, 2164 André, P., Ward-Thompson, D., \& Barsony, M. 1993, ApJ, 406, 122 André, P., Motte, F., \& Bacmann, A. 1999, ApJ, 513, L57 Bertoldi, F., \& McKee, C. F. 1992, ApJ, 395, 140

Bourke, T. L., Myers, P. C., Evans II, N. J., et al. 2006, ApJ, 649, L37 Condon, J. J., Cotton, W. D., Greisen, E. W., et al. 1998, AJ, 115, 1693 Dunham, M. L., Evans II, N. J., Bourke, T. L., et al. 2006, ApJ, 651, 945 Gomez, J. F., Trapero, J., Pascual, S., et al. 2000, MNRAS, 314, 743 Hearty, T., Neuhäuser, R., Stelzer, B., et al. 2000, A\&A, 353, 1044 Heithausen, A. 1999, A\&A, 345, L53

Heithausen, A., Bertoldi, F., \& Bensch, F. 2002, A\&A, 383, 591

Heithausen, A., Böttner, C., Walter, F. 2008, A\&A, 488, 597

Hobbs, L. M., Blitz, L., \& Magnani, L. 1986, ApJ, 306, 109

Johnstone, D., Fiege, J. D., Redman, R. O., Feldman, P. A., \& Carey, S. J. 2003 , ApJ, 588, L37

Kauffmann, J., Bertoldi, F., Evans II, N. J., et al. 2005, Astron. Nachr., 326, 878 Kauffmann, J., Bertoldi, F., Bourke, T. L., Evans II, N. J., \& Lee, C. W. 2008, A\&A, 487, 993

Littlefair, S. P., Dhillon, V. S., \& Marsh, T. R. 2001, MNRAS, 327, 669

Luhman, K. L. 2001, ApJ, 560, 287

Magnani, L., Blitz, L., \& Mundy, L. 1985, ApJ, 295, 402

Magnani, L., Caillault, J. P., Buchalter, A., \& Beichman, C. A. 1995, ApJS, 96, 159

Pound, M. W., Bania, T. M., \& Wilson, R. W. 1990, ApJ, 351, 165

Reach, W. T., Pound, M. W., Wilner, D. J., \& Lee, Y. 1995, ApJ, 441, 244

Rieke, G., Young, E. T., Engelbracht, C. W., et al. 2004, ApJS, 154, 25

Straižys, V., Černis, K., Kazlauskas, A., \& Laugalys, V. 2002, Baltic Astron., 11, 231

Werner, M., Roellig, T., Low, F., et al. 2004, ApJS, 154, 1

Zimmermann, T. 1993, Ph.D. Thesis, University of Cologne

Zimmermann, T., \& Ungerechts, H. 1990, A\&A, 238, 337 